\title{
Elastocaloric Effect Associated with the Martensitic Transition in Shape-Memory Alloys
}

\author{
Erell Bonnot, Ricardo Romero, ${ }^{*}$ Lluís Mañosa, Eduard Vives, and Antoni Planes \\ Departament d'Estructura i Constituents de la Matèria, Facultat de Física, Universitat de Barcelona, \\ Diagonal, 647, E-08028 Barcelona, Catalonia, Spain \\ (Received 19 October 2007; published 27 March 2008)
}

\begin{abstract}
The elastocaloric effect in the vicinity of the martensitic transition of a $\mathrm{Cu}-\mathrm{Zn}-\mathrm{Al}$ single crystal has been studied by inducing the transition by strain or stress measurements. While transition trajectories show significant differences, the entropy change associated with the whole transformation $\left(\Delta S_{t}\right)$ is coincident in both kinds of experiments since entropy production is small compared to $\Delta S_{t}$. The values agree with estimations based on the Clausius-Clapeyron equation. The possibility of using these materials for mechanical refrigeration is also discussed.
\end{abstract}

DOI: 10.1103/PhysRevLett.100.125901

Caloric effects are expected to occur under the application of an external field to a given material. The elastocaloric effect [1] is the mechanical analogue of the magnetocaloric effect that has received considerable attention in the recent years owing to its potential use for environmentally friendly refrigeration [2]. The magnetocaloric effect is related to the isothermal change of entropy or the adiabatic change of temperature that takes place within a material when a magnetic field is applied or removed. This effect originates from the coupling between the magnetic sublattice and an externally applied magnetic field and thus occurs in any magnetic material. A large effect is expected in the vicinity of field-induced, firstorder phase transitions where large entropy changes should occur [3]. By analogy, the elastocaloric effect is defined as the isothermal change of entropy or the adiabatic change of temperature that takes place when a mechanical field (stress) is applied or released in a given material. Indeed, this effect is expected to be a consequence of the coupling between an external applied stress and the lattice. Continuing with the analogy, a large elastocaloric effect is also foreseen in systems undergoing stress-induced, first-order phase transitions. Good candidates to show this effect are shape-memory alloys. These materials undergo a diffusionless purely structural transition from a cubic to a lower symmetry phase that can be stress induced [4]. Actually, shape-memory properties are related to this transition and refer to the ability of these systems to remember their original shape after severe deformation [5].

In contrast to magnetism, instead of controlling the applied stress (or force) which is the variable thermodynamically equivalent to the magnetic field, in mechanical experiments, the system is usually driven by controlling the strain (generalized displacement) which is the conjugated variable to the stress in the way that magnetization is the conjugated variable of the magnetic field. In magnetic systems, due to the difficulty in controlling magnetization, magnetization-driven experiments aimed at studying the magnetocaloric effect have not, to our knowledge, been reported. Thus, comparing results from both field- or stress-driven and magnetization or strain-driven experi-
PACS numbers: $65.40 . \mathrm{G}-$, 75.30.Sg, $81.30 . \mathrm{Kf}$

ments is of general interest since constraining the (generalized) displacement prevents free motion of the interfaces and therefore field/stress fluctuation can occur. Indeed, this is especially relevant in systems undergoing a phase transition leading to macroscopic instability. The study of mechanical systems naturally opens up the possibility of performing such kind of experiments. In a recent work [6] we have shown that metastable trajectories exhibit a strong dependence on the driving mechanism. In particular, strain-driven trajectories are characterized by the occurrence of reentrant behavior and lower dissipation than stress-driven trajectories.

The present Letter is aimed at studying the elastocaloric effect in the vicinity of the martensitic transition in a $\mathrm{Cu}_{68.13} \mathrm{Zn}_{15.74} \mathrm{Al}_{16.13}$ single crystal (molar volume $=$ $7.52 \mathrm{~cm}^{3} \mathrm{~mol}^{-1}$ ) in both the stress-driven and the straindriven modes. The sample was mechanically machined with cylindrical heads and the body has flat faces $35 \mathrm{~mm}$ long, $4 \mathrm{~mm}$ wide and $1.4 \mathrm{~mm}$ thick. Its axis is close to the [100] crystallographic direction of the cubic phase. This orientation provides high transformation strains for relatively low uniaxial applied tensile loads along this direction thus preventing the occurrence of irreversible plastic effects. The sample was conveniently heat treated to ensure that it was free from internal stresses and that the order state and vacancy concentration are close to their ground state values [6]. In the absence of any applied stress, the crystal undergoes a martensitic transition from a cubic $L 2_{1}$ phase to a multivariant $18 \mathrm{R}$ martensite at $T_{M}=234 \mathrm{~K}$.

For strain-driven experiments we used an Instron 4302 screw driven tensile machine in which the elongation is the control parameter. For stress-driven experiments we used a machine which enables control of the force applied to the sample while elongation was continuously monitored [6]. The machine applies a dead load to the sample which can be increased or decreased at a well controlled rate. A cryofurnace (with temperature stability $\pm 0.1 \mathrm{~K}$ ) can be adapted to both devices. All experiments have been performed at low rates $\left[\sim 0.3 \mathrm{~mm} \mathrm{~min}^{-1}\right.$ (strain-driven) and $\sim 5 \mathrm{MPa} \mathrm{min}^{-1}$ (stress-driven)]. 
Let us now consider a thermodynamic system described by variables $\{\mathbf{X}, \mathbf{Y}, T\}$, where $\mathbf{X}$ is a generalized displacement and $\mathbf{Y}$ is the corresponding conjugated field ( $\mathbf{X}$ and $\mathbf{Y}$ have the same tensorial order), and $T$ is the temperature. A change in the generalized displacement gives rise to a caloric effect. If this change is induced by an isothermal change $\Delta \mathbf{Y}$ of its conjugated field, the caloric effect must be quantified by the corresponding induced entropy change which is given by

$$
\Delta S=\int_{\Delta \mathbf{Y}}\left(\frac{\partial \mathbf{X}}{\partial T}\right)_{\mathbf{Y}} d \mathbf{Y}
$$

where the generalized Maxwell relation $(\partial S / \partial \mathbf{Y})_{T}=$ $(\partial \mathbf{X} / \partial T)_{\mathbf{Y}}$ has been taken into account. When the field is an uniaxial tensile stress $\sigma$ for which the corresponding strain (or relative elongation along the direction of the applied force) is $\varepsilon$, the induced isothermal entropy change defining the elastocaloric effect, is given by

$$
\Delta S(0 \rightarrow \sigma)=\int_{0}^{\sigma}\left(\frac{\partial \varepsilon}{\partial T}\right)_{\sigma} d \sigma
$$

This expression is formally analogous to the expression giving the field-induced entropy change which defines the magnetocaloric effect in a magnetic system [2]. If instead of $\sigma$ the controlled variable is $\varepsilon$, the entropy change corresponding to an isothermal variation of the strain from 0 to $\varepsilon$ is given by

$$
\Delta S(0 \rightarrow \varepsilon)=-\int_{0}^{\varepsilon}\left(\frac{\partial \sigma}{\partial T}\right)_{\varepsilon} d \varepsilon,
$$

where the Maxwell relation $(\partial S / \partial \varepsilon)_{T}=-(\partial \sigma / \partial T)_{\varepsilon}$ has been used in this case. Of course, if $\varepsilon$ is the strain corresponding to the stress $\sigma$, in strict equilibrium $\Delta S(0 \rightarrow$ $\sigma)=\Delta S(0 \rightarrow \varepsilon)$.

Now assume a system subjected to an applied uniaxial stress $\sigma$ that undergoes a first-order structural (martensitic) phase transition in equilibrium at a temperature $T_{t}$. The transition is in this case characterized by discontinuities in variables such as strain and entropy that are thermodynamically conjugated to the intensive variables stress and temperature. In the vicinity of the transition the following behavior of the strain is thus expected, $\varepsilon(T, \sigma)=\varepsilon_{0}+$ $\Delta \varepsilon \mathcal{F}\left[\left(T_{t}(\sigma)-T\right) / \Delta T\right]$, where $\mathcal{F}$ is a shape-function and $\Delta T$ is a measure of the temperature range over which the transition spreads. In strict equilibrium, $\Delta T \rightarrow 0$ so that $\mathcal{F}$ approaches the Heaviside step function. Using expression (2) and assuming that $\varepsilon_{0}$ and $\Delta \varepsilon$ are constant, in this equilibrium case the elastocaloric effect in the vicinity of the transition is given by

$$
\Delta S(0 \rightarrow \sigma)= \begin{cases}-\frac{\Delta \varepsilon}{\alpha} & \text { for } T \in\left[T_{t}(0), T_{t}(\sigma)\right] \\ 0 & \text { for } T\left[T_{t}(0), T_{t}(\sigma)\right]\end{cases}
$$

where $\alpha \equiv d T_{t} / d \sigma$ is assumed to be constant. Taking into account the Clausius-Clapeyron equation, $\alpha=-\Delta \varepsilon / \Delta S_{t}$, where $\Delta S_{t}$ is the transition entropy change. Therefore, as expected, $\Delta S(0 \rightarrow \sigma)=\Delta S_{t}$, and $\Delta T=T_{\sigma}-T_{t}(0)=$ $\alpha \sigma$. Indeed, the same result is obtained in this case from Eq. (3).

Actually, these transitions are spread over a small range of $\sigma$, and, more importantly, they display hysteresis, which reflects the existence of nonequilibrium dissipative effects. In this case, taking into account the Clausius inequality, $\oint \delta q / T \leq 0$, for an isothermal process, the entropy change must satisfy, $\Delta S(0 \rightarrow \sigma$ or $\varepsilon)=\frac{q}{T}+S_{i}$, where $S_{i} \geq 0$ is the entropy production and is expected to depend on the actual trajectory followed by the system (strain- or stressdriven in our case). The preceding equation indicates that an estimation of the entropy change based on expression (2) should differ from an estimation based on direct calorimetric measurements. For the system of interest here, as hysteresis is quite small, estimations of the entropy change based on stress-driven and strain-driven curves should provide reasonably good estimations of the elastocaloric effect.

In Fig. 1 we show stress-strain curves obtained in the stress-driven case at selected temperatures (well above the transition temperature at zero-stress) across the martensitic transition in the studied $\mathrm{Cu}-\mathrm{Zn}-\mathrm{Al}$ single crystal. The shift of the transition to higher stresses with increasing temperature is clearly seen. Comparison of curves corresponding to loading and unloading shows that the transition occurs with weak hysteresis of about $10 \mathrm{MPa}$. From these curves the elastocaloric effect (stress-induced entropy change) has been obtained by numerically computing the integral in Eq. (2). The stress-induced entropy change (elastocaloric effect) is shown in Fig. 2.

It is interesting to point out that the maximum stressinduced entropy change (which corresponds to the whole

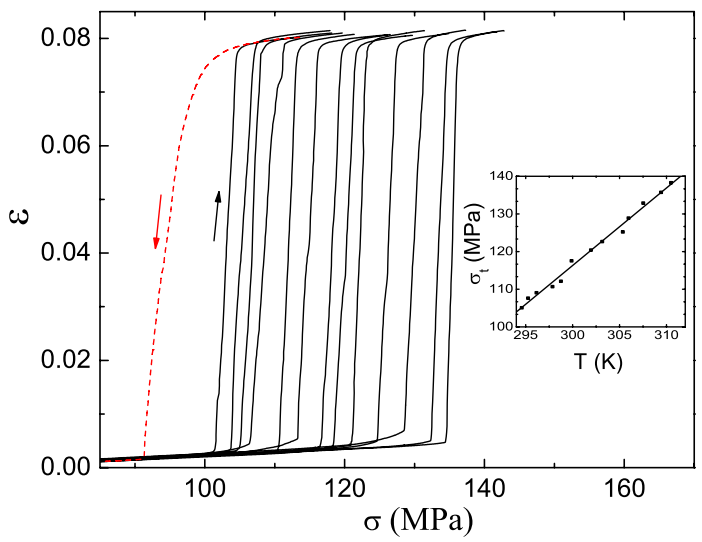

FIG. 1 (color online). Stress-strain curves at selected temperatures. Continuous curves correspond to loading runs (from right to left) at $T=310.5,309.4,307.5,306.0,305.4,303.1,302.0$, 299.9, 298.8, 297.9, 296.1, 295.3, and 294.6 K. The left discontinuous curve corresponds to the unloading branch at $T=$ 294.6 K. It illustrates an example of stress-induced hysteresis loops. The inset shows the transition stress as a function of temperature. The line is a linear fit $\sigma=2.01(T-242)$. 


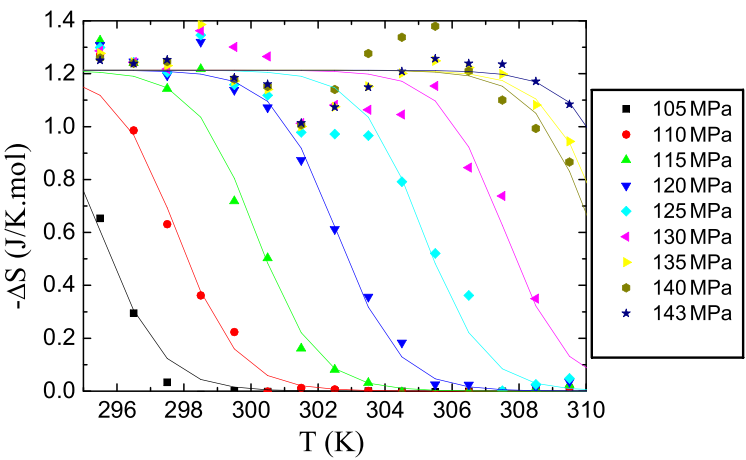

FIG. 2 (color online). Stress-induced entropy change (elastocaloric effect) at selected values of $\sigma$ ranging from 105 to $143 \mathrm{MPa}$. The continuous lines are fits based on the model $\mathcal{F}(x)=\tanh ^{-1}(x)$.

entropy change of the martensitic transformation) remains almost constant (thus independent of temperature and applied stress) over a very broad temperature range [7]. This is usually not the case for the magnetocaloric effect in field-induced, first-order phase transitions for which large $\Delta S(T)$ is only obtained in a relatively narrow temperature interval (which depends on the applied field). Such a difference is a consequence of the fact that here tensile experiments are performed at temperatures well above ( $\geq$ $60 \mathrm{~K})$ the transition temperature at zero stress. This is possible due to the strong dependence of the transition stress with temperature. The upper bound is imposed by the elastic limit of the cubic phase. By contrast, in magnetic experiments the field is always applied close to the transition temperature at zero field. Indeed, at higher temperatures metamagnetic transitions are difficult to be induced either because intense magnetic fields that are too large are required or simply because this leads the system above the critical point, where no transition occurs. The continuous lines in Fig. 2 are fits which assume a shapefunction $\mathcal{F}(x)=\tanh ^{-1}(x)$. In the strain-driven case we can proceed similarly starting with strain-stress curves recorded at selected temperatures. Figure 3 displays the strain-stress curves obtained at selected temperatures. The strain-induced entropy change (elastocaloric effect) as a function of $T$ has then been obtained using Eq. (3). Results are shown in Fig. 4. The variation of $\Delta S$ with temperature reflects a small variation of $\Delta \varepsilon$ with temperature.

From the previous results the estimated [8] entropy change corresponding to the whole transition is $\Delta S_{t}=$ $-1.20 \pm 0.15 \mathrm{~J} / \mathrm{mol} \mathrm{K}$ consistent in both strain- and stress-driven cases. It is interesting to compare this result with estimations based on the use of the ClausiusClapeyron equation. From the stress-driven curves in Fig. 1 we can define a transition stress, $\sigma_{t}$, at each temperature, as the stress at the inflection point of the isotherms. The strain change, $\Delta \varepsilon$, at the transition can also be estimated from these curves. The transition entropy change is then obtained as $\Delta S_{t} \simeq\left(d \sigma_{t} / d T\right) \Delta \varepsilon$. In the inset of

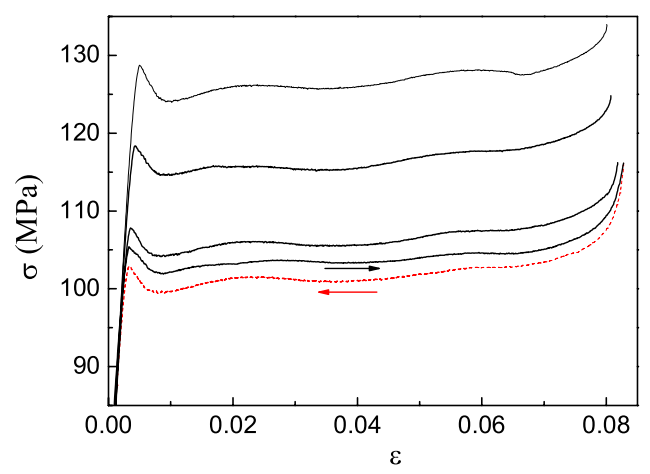

FIG. 3 (color online). Strain-stress curves obtained at selected temperatures. Continuous curves correspond to loading runs at (from top to bottom): $T=307.8,303.1,297.8$, and $295.0 \mathrm{~K}$. The lower discontinuous curve corresponds to the unloading branch at $T=295.0 \mathrm{~K}$. It illustrates an example of a strain-induced hysteresis loop.

Fig. 1 we show $\sigma_{t}$ vs $T$. We see that $\sigma_{t}$ rises linearly with increasing $T$, with a slope $d \sigma_{t} / d T=2.01 \mathrm{MPa} / \mathrm{K}$. Taking an average value $\Delta \varepsilon=0.080 \pm 0.005$ we obtain $\Delta S_{t}=-1.21 \pm 0.05 \mathrm{~J} / \mathrm{mol} \mathrm{K}$. This value is compatible with the estimations based on elastocaloric effect. It is interesting to compare this value with a calorimetric measurement. To this end, we have carried out calorimetric measurements of the transition entropy change at zero stress, using a small specimen cut from the same original ingot. These experiments give a value $\Delta S_{t}=-1.37 \pm$ $0.10 \mathrm{~J} / \mathrm{mol} \mathrm{K}$, slightly higher (as an absolute value) than that derived from mechanical experiments. Such a difference should not, in principle, be attributed to nonequilibrium effects which have been estimated from the area of the hysteresis loops to be of the order of $0.01 \mathrm{~J} / \mathrm{mol} \mathrm{K}$ in the stress-induced case and less than $0.001 \mathrm{~J} / \mathrm{mol} \mathrm{K}$ in strain-induced experiments. However, it must be pointed out that calorimetric measurements are performed in the absence of an external field and thus a multivariant martensite is reached. Kinematic constraints which occur during the transformation and yield extra dissipative effects could explain the difference. This is consistent with the fact

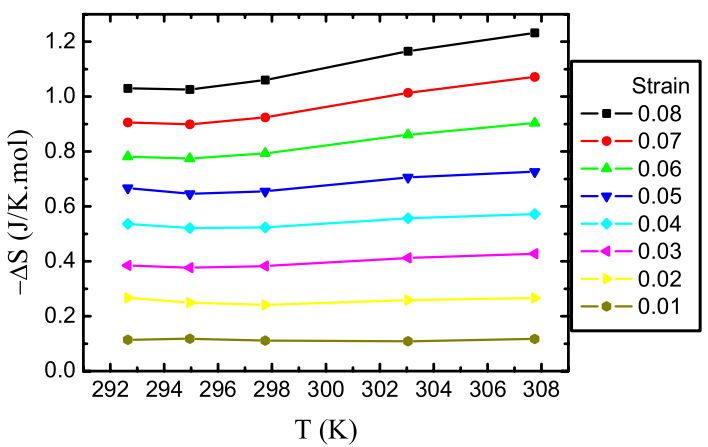

FIG. 4 (color online). Strain-induced entropy change (elastocaloric effect) at selected values of $\epsilon$ from 0.01 to 0.08 . 
that the transition temperature in the multivariant case $(234 \mathrm{~K})$ is significantly lower than the extrapolation to zero stress of the $\sigma$ vs $T$ curve $(242 \mathrm{~K}$ ) which corresponds to the transition to a single variant martensite (see inset of Fig. 1).

The elastocaloric effect has been reported in the Fe-Rh alloy [9]. In this case the entropy change has a magnetic origin associated with a reorientation of spins taking place at the first-order antiferromagnetic to ferromagnetic magnetostructural transition which is induced by the application of a tensile stress due to strong magnetoelastic coupling. Similar effects (termed the barocaloric effect) have been reported for a number of rare-earth compounds subjected to uniaxial pressure [10]. In the present study, however, the elastocaloric effect is associated with a purely structural transition. In this case, the entropy change is predominantly vibrational and originates from the very low energy $\mathrm{TA}_{2}$ transverse ([110] propagation and [110] polarization) phonon branch of the cubic phase [4]. It is also worth noticing that in Fe-Rh the entropy change is positive when the stress is isothermally applied, and thus the sample cools down when the stress is adiabatically applied. This corresponds to an inverse elastocaloric effect which is the analog of the inverse magnetocaloric effect reported in Heusler martensitic alloys [11]. Notice that inverse effects are only possible when there is strong coupling between magnetic and structural degrees of freedom.

The adiabatic temperature change associated with the elastocaloric effect can be estimated as $\Delta T \simeq-\frac{T}{C} \Delta S$, where $C$ is the specific heat which is assumed to be stress independent. For $\mathrm{Cu}-\mathrm{Zn}-\mathrm{Al}$, the value for the specific heat close to room temperature is approximately $25 \mathrm{~J} / \mathrm{K} \mathrm{mol}$ in both martensitic and cubic phases [12]. For an adiabatic drop involving the whole transition, the maximum expected temperature change is $15 \mathrm{~K}$. Notice that this value is orders of magnitude larger than the typical values in elastic solids far from any phase transition. The effect of strain rate on the martensitic transition of several $\mathrm{Cu}$-based shape-memory alloys was studied by means of direct measurements of the temperature changes associated with the transition. For $\mathrm{Cu}-\mathrm{Al}-\mathrm{Ni}$ [13] and $\mathrm{Cu}-\mathrm{Zn}-\mathrm{Sn}$ [14] crystals (with the same martensitic structure as our $\mathrm{Cu}-\mathrm{Zn}$-Al crystal), the measured temperature changes at high strain rates (close to the adiabatic limit) are 14 and $12 \mathrm{~K}$, respectively, for a strain rate of $25 \mathrm{~min}^{-1}$. These temperature values compare well to the value indirectly computed here for $\mathrm{Cu}$ $\mathrm{Zn}-\mathrm{Al}$. On the other hand, present values for $\mathrm{Cu}-\mathrm{Zn}-\mathrm{Al}$ are comparable to those reported for other elastocaloric materials undergoing first-order magnetostructural phase transitions: maximum changes of 8.7 and $14 \mathrm{~K}$ are computed for Fe-Rh [9] and Eu-Ni-Si-Ge [10], respectively.

The elastocaloric effect associated with the martensitic transition in a $\mathrm{Cu}-\mathrm{Zn}-\mathrm{Al}$ single crystal has been studied. It is formally equivalent to the magnetocaloric effect in a magnetic system and in our case it should be comparable to the magnetocaloric effect in the vicinity of a first-order metamagnetic transition. However, while the magnetocaloric effect is always determined by the field inducing the metamagnetic transition, for the mechanical case it has been possible to obtain the isothermal entropy change by inducing the structural transition using both strain and stress. While the transition path is essentially different in both cases, the corresponding isothermal entropy changes are the same to within errors. This is due to the fact that hysteresis is small independent of the driving mechanism. For practical applications of caloric effects, the refrigerant capacity is a central parameter to be considered [3]. It is defined as $\mathcal{R}=\int_{\Delta T} \Delta S(T) d T \simeq \Delta S \Delta T=-\Delta \varepsilon \Delta \sigma$, where $\Delta \sigma$ is the change of $\sigma$ necessary to change the transition temperature by $\Delta T(\Delta S$ and $\Delta \varepsilon$ are assumed constants). The interest in our case is that $\Delta \sigma$ can be chosen in a broad range of values which opens up interesting opportunities in refrigeration applications based on the elastocaloric effect.

This work received financial support from CICyT (Spain), Project No. MAT2007-61200, Marie-Curie RTN MULTIMAT (EU), Contract No. MRTN-CT-2004-505226, and DURSI (Catalonia), Project No. 2005SGR00969. The authors acknowledge O. Toscano for experimental assistance.

*Permanent address: IFIMAT, Universidad del Centro de la Provincia de Buenos Aires, Pinto, 399, 7000 Tandil, Argentina.

[1] This effect is also termed piezocaloric effect. See for instance, J.F. Nye, Physical Properties of Crystals (Oxford University, New York, 1957).

[2] A.M. Tishin and Y.I. Spichkin, The Magnetocaloric Effect and its Applications (Institute of Physics, Bristol and Philadelphia, 2003); E. Brück, J. Phys. D 38, R381 (2005).

[3] K. A. Gschneidner, V. K. Pecharsky, and A. O. Tsokol, Rep. Prog. Phys. 68, 1479 (2005).

[4] A. Planes and Ll. Mañosa, in Solid State Physics, Vol. 55 (Academic, New York, 2001), p. 159.

[5] K. Otsuka and C.M. Wayman, in Shape Memory Materials, edited by K. Otsuka and C.M. Wayman (Cambridge University Press, Cambridge, 1998), p. 1.

[6] E. Bonnot et al., Phys. Rev. B 76, 064105 (2007).

[7] The isothermal entropy change will significantly decrease below the martensitic transition temperature at zero stress.

[8] Error limits are based on reproducibility of experiments.

[9] S. A. Nikitin et al., Phys. Lett. A 171, 234 (1992); M. P. Annaorazov et al., J. Appl. Phys. 79, 1689 (1996).

[10] Th. Strässle et al., Phys. Rev. B 67, 054407 (2003).

[11] T. Krenke et al., Nat. Mater. 4, 450 (2005).

[12] J. C. Lashley et al., Phys. Rev. B 75, 064304 (2007).

[13] C. Rodríguez, L. C. Brown, Metall. Trans. A 11, 147 (1980).

[14] L. C. Brown, Metall. Trans. A 12, 1491 (1981). 